\title{
Magnetic Anisotropy, Spin Pinning and Exchange Constants of (Ga,Mn)As films
}

\author{
Ying-Yuan Zhou, Yong-Jin Cho, Zhiguo Ge, Xinyu Liu, Malgorzata Dobrowolska, and Jacek K. Furdyna \\ Department of Physics, University of Notre Dame, Notre Dame, IN 46556, USA
}

\begin{abstract}
We present a detailed investigation of exchange-dominated nonpropagating spin-wave modes in a series of $100 \mathrm{~nm} \mathrm{Ga}{ }_{-x} \mathrm{Mn}_{x}$ As films with $\mathrm{Mn}$ concentrations $x$ ranging from 0.02 to 0.08 . The angular and $\mathrm{Mn}$ concentration dependences of spin wave resonance modes have been studied for both as-grown and annealed samples. Our results indicate that the magnetic anisotropy terms of $\mathrm{Ga}_{1-x} \mathrm{Mn}_{x}$ As depend on the Mn concentration $x$, but are also strongly affected by sample growth conditions; moreover, the magnetic anisotropy of $\mathrm{Ga}_{1-x} \mathrm{Mn}_{x}$ As films is found to be clearly linked to the Curie temperature. The spin wave resonance spectra consist of a series of well resolved standing spin-wave modes. The observed mode patterns are consistent with the Portis volume-inhomogeneity model, in which a spatially nonuniform anisotropy field acts on the Mn spins. The analysis of these exchange-dominated spin wave modes, including their angular dependences, allows us to establish the exchange stiffness constants for $\mathrm{Ga}_{1-x} \mathrm{Mn}_{x}$ As films.
\end{abstract}

PACS numbers:

Keywords:

\section{INTRODUCTION}

Incorporating $\mathrm{Mn}$ ions into III-V semiconductors makes it possible to achieve ferromagnetic order in semiconductor nanostructures 1 . It is known that the spin waves in a magnetic system are determined by (and can be used to obtain) the exchange and magnetic anisotropy parameters $\mathbf{2}^{2,3,4,5,6}$, and are thus particularly useful for a quantitative understanding of ferromagnetism in $\mathrm{Ga}_{1-x} \mathrm{Mn}_{x} \mathrm{As}^{2}$. In this paper we use ferromagnetic resonance (FMR), a powerful tool for investigating magnetic anisotropy and exchange constants in ferromagnetic materials ${ }^{7}$, to investigate a series of $\mathrm{Ga}_{1-x} \mathrm{Mn}_{x} \mathrm{As}$ films with different $\mathrm{Mn}$ concentrations $x$. In all samples studied we observe a multi-mode spin wave resonance (SWR) spectrum. We will focus on the detailed description of such SWRs, and the relationship between these spin wave modes and the Mn concentration $x$ in $\mathrm{Ga}_{1-x} \mathrm{Mn}_{x}$ As.

\section{SAMPLE FABRICATION}

A series of $\mathrm{Ga}_{1-x} \mathrm{Mn}_{x}$ As films were grown by molecular beam epitaxy (MBE) on semi-insulating GaAs (001) substrates. A $100 \mathrm{~nm}$-thick GaAs buffer was first grown at the substrate temperature $600^{\circ} \mathrm{C}$ to achieve an atomically flat surface. The substrates were then cooled to $250^{\circ} \mathrm{C}$ for growth of a $2 \mathrm{~nm}$ low temperature GaAs buffer, followed by $100 \mathrm{~nm} \mathrm{Ga}{ }_{1-x} \mathrm{Mn}_{x}$ As layers with various $\mathrm{Mn}$ concentrations $(x=0.02,0.025,0.04,0.045,0.055,0.06$, 0.07 and 0.08$)$. The thickness of $(\mathrm{Ga}, \mathrm{Mn}) \mathrm{As}$ samples is specifically selected to reliably determine the effect of annealing on SWRs. All as-grown specimens exhibited ferromagnetic order, with Curie temperature $T_{C}$ ranging from $50 \mathrm{~K}$ to $80 \mathrm{~K}$. Pieces of samples cleaved from each specimen were then annealed in $\mathrm{N}_{2}$ gas for one hour at $280^{\circ} \mathrm{C}$ in order to examine the effect of annealing on magnetic properties. As a result of annealing $T_{C}$ of the sam- ples increased to a range from $55 \mathrm{~K}$ to $115 \mathrm{~K}$. Note that $T_{C}$ follows a general rule for both as-grown and annealed samples: it increases with increasing concentration $x$.

\section{EXPERIMENTAL SETUP}

In this study we have measured the angular dependence of FMR for each specimen (both as-grown and annealed) in three geometries ${ }^{7}$. FMR measurements were carried out at $9.46 \mathrm{GHz}$ using a Bruker electron paramagnetic resonance spectrometer. The applied dc magnetic field $\mathbf{H}$ was in the horizontal plane, while the microwave magnetic field was acting vertically on the sample. The sample was placed in a suprasil tube inserted in a liquid helium continuous flow cryostat, which could achieve temperatures down to $4.0 \mathrm{~K}$.

The $\mathrm{Ga}_{1-x} \mathrm{Mn}_{x}$ As layers were cleaved into three square pieces with edges along the [110] and [1̄0] directions. Each square piece was then placed in three different orientations. Geometry 1 is when the sample plane and the

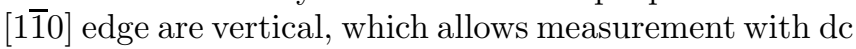
magnetic field $\mathbf{H}$ oriented at any angle between $\mathbf{H} \|[001]$ (normal orientation) and $\mathbf{H} \|[110]$ (in plane orientation). Geometry 2 is when the sample plane and the [010] direction are vertical, which allows us to measure FMR with field orientations between $\mathbf{H} \|[001]$ and the in-plane orientation $\mathbf{H} \|[100]$. Geometry 3 is when the sample plane is horizontal, allowing us to map out the FMR when $\mathbf{H}$ is confined to the layer plane.

\section{RESULTS AND DISCUSSIONS}

\section{A. Magnetic Anisotropy}

Multi-mode SWR spectrum was observed in all samples. Our analysis of the data was carried out as follows. We first obtained the magnetic anisotropies and $g$-factors 


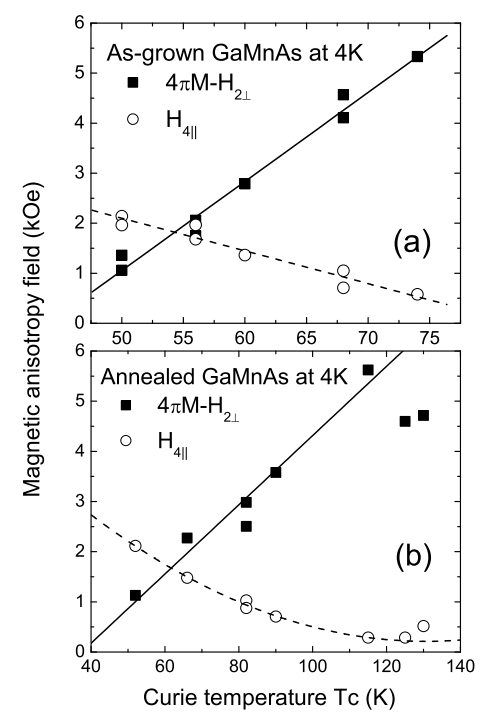

FIG. 1: The effective anisotropy term $4 \pi M-H_{2 \perp}$ and the in-plane cubic anisotropy $H_{4 \|}$ versus the Curie temperature of (a) as-grown samples, and (b) annealed samples at $\mathrm{T}=4$ $\mathrm{K}$. The solid and dashed lines are guides for the eye.

by fitting the angular dependence of the strongest resonance line to a theoretical uniform FMR model using a nonlinear least squares method ${ }^{8}$. Our fitting results show that the magnitude of perpendicular uniaxial anisotropy $H_{2 \perp}$ has tends to increase with Mn concentration $x$, while the in-plane cubic anisotropy $H_{4 \|}$ decreases with $x$. However, the relation between the magnetic anisotropy terms and $x$ is strongly influenced by the individual growth condition of each sample. Furthermore, we find that the term $4 \pi M_{e f f}=4 \pi M-H_{2 \perp} \underline{\underline{3}}$ is linearly increasing with the Curie temperature $T_{C}$ in both as-grown and annealed specimens, and $H_{4 \|}$ is monotonically decreasing with $T_{C}$, with the exception of annealed specimens with the highest values of $T_{C}$. The results are shown in Fig. 1. Since an earlier report $\frac{9}{9}$ shows an empirical relationship $T_{C} \sim p^{1 / 3}$, we immediately note that there exists a tight relationship between magnetic anisotropy and the hole concentration $p$. However, the data for as-grown and annealed samples follow different slopes as a function of $T_{C}$, suggesting that there exists a fundamental differences between as-grown and annealed samples regarding magnetic anisotropy. Nevertheless, the distinct characteristics which we observe may provide useful insights into the origin of magnetic anisotropy in $\mathrm{Ga}_{1-x} \mathrm{Mn}_{x} \mathrm{As}$ films.

\section{B. SWR Spectra}

It is the spin pinning at sample surfaces that induces spin-wave excitation in the FMR experiment. To investi-

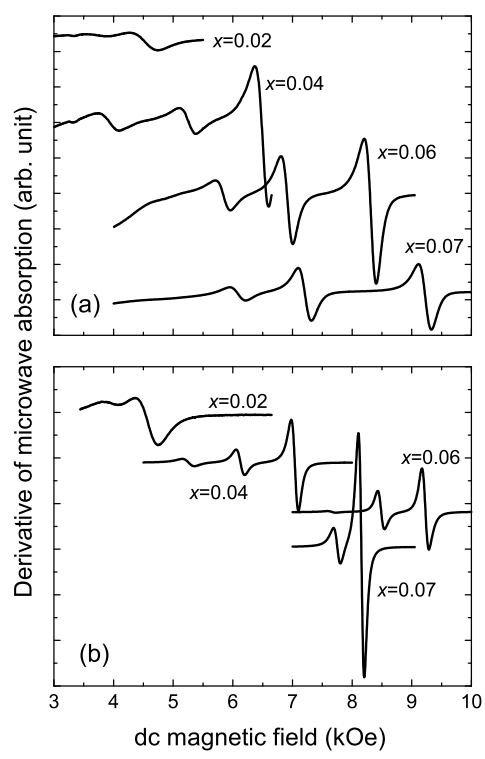

FIG. 2: The SWR spectra at $4 \mathrm{~K}$ when the external dc magnetic field is perpendicular to the film $(\mathbf{H} \|[001])$ for (a) asgrown samples and (b) annealed samples.

gate this feature in detail, we have chosen four specimens grown at similar conditions, with Mn concentration $x=$ $0.02,0.04,0.06$ and 0.07 . Figure 2 shows the SWR spectra at $\mathrm{T}=4 \mathrm{~K}$ for both as-grown and annealed samples when the dc magnetic field $\mathbf{H}$ is normal to the sample plane $\left(\mathbf{H} \|[001]\right.$, i.e., $\left.\theta_{H}=0^{\circ}\right)$. In both cases the SWR spectra consist of several well-resolved standing spin wave modes separated by roughly equal field increments. Note that the SWR fields are increasing with the Mn concentration $x$, except in the case of one annealed sample with Mn concentration $x=0.07$. Importantly, the as-grown samples have a larger mode separation than the annealed samples, which indicates that the exchange stiffness constant $D$ is larger in the as-grown samples, as discussed later.

As $\mathbf{H}$ is rotated away from the perpendicular orientation, the low-field spin wave modes gradually disappear, and eventually only one narrow resonance line remains at a critical angle $\theta_{c}$. We will refer to it as the uniform mode. For angle $\theta_{H}>\theta_{c}$ the spectrum generally consists of two wide resonance lines. We identify the mode lying at the higher field as an exchange-dominated surface spin wave mode 10,11 . The angular dependence of the SWRs reveals the nature of surface spin pinning and its dependence on the magnetization orientation. Using the Puszkarski surface inhomogeneity (SI) mode ${ }^{10}$, it is found that, as the magnetization rotates from the perpendicular to the in-plane orientation, the surface spins are evolving from a strong pinning condition to a weak pinning condition, with a turning point at the critical 
angle $\theta_{c}{ }_{c}^{11}$. Note that for $\mathbf{H} \|[001]$ the pinning is not only strong, but also nonlocalized, i.e., the magnetic spins appear to be affected by a spacially nonuniform anisotropy field $^{12}$, suggested by a linear mode separation at this orientation.

\section{Exchange Constants}

We will now focus on the SWR spectrum obtained for $\mathbf{H} \|[001]$. It is found that the positions of the SWR modes comply with a linear mode separation model for $\mathbf{H} \|[001]$ when a symmetrical parabolic magnetic anisotropy is assumed along the growth direction $z(|z| \leq L / 2)$ : $4 \pi M^{*}(z)=4 \pi M^{*}(0)\left(1-4 \varepsilon z^{2} / L\right) \underline{13}$. Here $\varepsilon$ is the distortion parameter of the film, $L=100 \mathrm{~nm}$ is the film thickness, and $4 \pi M^{*}(0)=4 \pi M-H_{2 \perp}-H_{4 \perp}$. In this situation the position of the $n$-th SWR mode for $\mathbf{H} \|[001]$ is given by the Portis relation ${ }^{12}$ :

$$
H_{n}=H_{0}-\left(n-\frac{1}{2}\right)(4 / L)\left(4 \pi M^{*}(0) \varepsilon \frac{D}{g \mu_{B}}\right)^{1 / 2},
$$

where $\mu_{B}$ is the Bohr magneton, $n$ is an odd integer, and $H_{0}=\omega / \gamma+4 \pi M^{*}(0)$ is the position of the theoretical uniform mode. Here $\omega$ is the angular frequency of the microwave field and $\gamma$ is the gyromagnetic ratio. The exchange stiffness constant $D$, which gives a measure of the strength of the exchange interaction, can be determined from the difference of the adjacent SWR modes by:

$$
\frac{D}{g \mu_{B}}=\frac{\Delta H_{n 1, n 2}^{2} L^{2}}{16(n 2-n 1)^{2}\left(4 \pi M^{*}(0) \varepsilon\right)} .
$$

Note that the value of $4 \pi M^{*}(0) \varepsilon$, the depth of parabolic potential well, can be roughly evaluated from the field separation between the highest and the lowest SWR modes observed for $\mathbf{H} \|[001]^{11}$. In the special case where only two modes are observed (see Fig. $2, x=0.07$ ), it is interesting that $\Delta H_{1,3} \approx 4 \pi M^{*}(0) \varepsilon$, so that $D$ can be satisfactorily estimated simply from the separation of the two modes.

Using Eq. (2), we can obtain the value of $D / g \mu_{B}$ for all samples. The data for four optimally-grown samples are plotted as function of Mn concentration $x$ in Fig. 3. The figure clearly shows that $D$ is closely related to $x$. In particular, for as-grown samples $D$ decreases very quickly as $x$ increases. On the other hand, although the data for annealed samples suggest that $D$ also decreases as $x$ increases, the change is much smaller in this case. The most important characteristic shown in Fig. 3 is that $D$ decreases after annealing, especially for low Mn concentration samples. This experimental result is quite surprising, because $T_{C}$ - which usually go hand-in-hand with the strength of the exchange interaction - are higher in the annealed samples than in the as-grown films. One possible explanation is that the average distance between

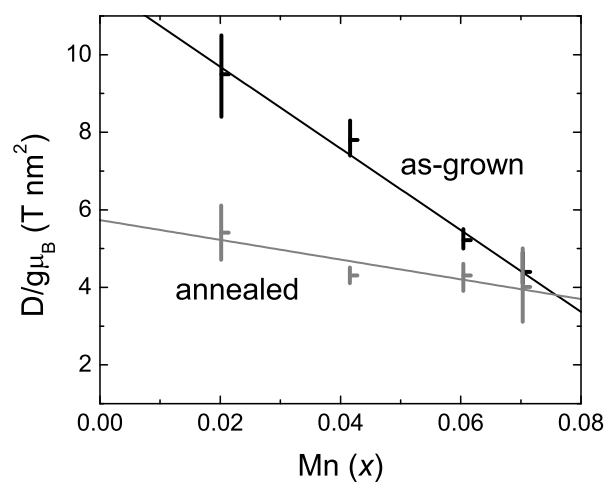

FIG. 3: The exchange stiffness constant $D$ as a function of Mn concentration $x$ for as-grown and annealed GaMnAs films. The lines are linear fits.

substitutional Mn ions (which links $T_{C}$ to $D$ ) is changed by the annealing process sufficiently to cause such a result. Indeed, significant changes in the lattice parameter of (Ga,Mn)As have been observed experimentally. However, the exact mechanism of such behavior is not understood and requires further study.

\section{SUMMARY AND CONCLUSIONS}

In summary, we carried out a detailed experimental study of SWRs in thin $\mathrm{Ga}_{1-x} \mathrm{Mn}_{x}$ As films with a wide range of $\mathrm{Mn}$ concentrations $x$. The analysis of the data allowed us to establish the following important magnetic properties of these films: magnetic anisotropy, surface spin pinning, and the exchange stiffness constant. The magnetic parameters obtained from this analysis were studied as function of $x$ and/or $T_{C}$ of the specimens. We observe a significant change of magnetic properties between as-grown and annealed samples. The results clearly show that the study of SWRs provides valuable information about the strong correlation between structural and magnetic properties of these materials.

\section{Acknowledgment}

This work was supported by the NSF Grant DMR0603752 .
1 H. Ohno, "Making nonmagnetic semiconductors ferromagnetic," Science, vol. 281, pp. 951-956, 1998.
2 J. König, T. Jungwirth, and A. H. MacDonald, "Theory 
of magnetic properties and spin-wave dispersion for ferromagnetic (Ga,Mn)As," Phys. Rev. B, vol. 64, pp. 184423, 2001.

3 X. Liu, Y. Sasaki, and J. K. Furdyna, "Ferromagnetic resonance in $\mathrm{Ga}_{1-x} \mathrm{Mn}_{x}$ As: Effects of magnetic anisotropy," Phys. Rev. B, vol. 67, pp. 205204, 2003.

4 T. G. Rappoport, P. Redliski, X. Liu, G. Zaránd, J. K. Furdyna, and B. Jankó, "Anomalous behavior of spin-wave resonances in $\mathrm{Ga}_{1-x} \mathrm{Mn}_{x}$ As thin films," Phys. Rev. B, vol. 69, pp. 125213, 2004.

5 S. T. B. Goennenwein, T. Graf, T. Wassner, M. S. Brandt, M. Stutzmann, J. B. Philipp, R. Gross, M. Krieger, K. Zürn, P. Ziemann, A. Koeder, S. Frank, W. Schoch, and A. Waag, "Ferromagnetic Resonance in $\mathrm{Ga}_{1-x} \mathrm{Mn}_{x} \mathrm{As}$," $J$. Supercond., vol. 16, pp. 75, 2003.

${ }^{6}$ S. T. B. Goennenwein, T. Graf, T. Wassner, M. S. Brandt, M. Stutzmann, A. Koeder, S. Frank, W. Schoch and A. Waag, "Spin wave resonance in $\mathrm{Ga}_{1-x} \mathrm{Mn}_{x} \mathrm{As}$," Appl. Phys. Lett., vol. 82, pp. 730, 2003.

7 X. Liu and J. K. Furdyna, "Ferromagnetic resonance in $\mathrm{Ga}_{1-x} \mathrm{Mn}_{x}$ As dilute magnetic semiconductors," J. Phys.: Condens. Matter, vol. 18, pp. R245-R279, 2006.
8 X. Liu, W. L. Lim, L. V. Titova, M. Dobrowolska, J. K. Furdyna, M. Kutrowski, and T. Wojtowicz, "Perpendicular magnetization reversal, magnetic anisotropy, multistep spin switching, and domain nucleation and expansion in $\mathrm{Ga}_{1-x} \mathrm{Mn}_{x}$ As films," J. Appl. Phys., vol. 98, pp. 63904, 2005.

9 A. H. MacDonald, P. Schiffer, and N. Samarth, "Ferromagnetic semiconductors: moving beyond (Ga,Mn)As," Nature Materials, vol. 4, pp. 195-202, 2005.

10 H. Puszkarski, "Theory of surface states in spin wave resonance," Prog. Surf. Sci., vol. 9, pp. 191-247, 1979.

11 X. Liu, Y. Y. Zhou, and J. K. Furdyna, "Angular Dependence of Spin Wave Resonances and Surface Spin Pinning in Ferromagnetic (Ga,Mn)As Films," Phys. Rev. B, submitted.

12 A. M. Portis, "Low-lying spin wave modes in ferromagnetic films," Appl. Phys. Lett., vol. 2, pp. 69-71, 1963.

13 Y. Sasaki, X. Liu, T. Wojtowicz, and J. K. Furdyna, "Spin wave resonances in GaMnAs," J. Supercond., vol. 16, pp. 143-145, 2003. 